\title{
Fountains, histograms, and q-identities
}

\author{
Peter Paule ${ }^{1}||^{i}$ and Helmut Prodinger2非
}

\author{
${ }^{1}$ Research Institute for Symbolic Computation, Johannes Kepler University Linz, A-4040 Linz, Austria \\ e-mail: Peter.Paule@risc.uni-linz.ac.at \\ Url: http://www.risc.uni-linz.ac.at/research/combinat/ \\ 2 The John Knopfmacher Centre for Applicable Analysis and Number Theory, School of Mathematics, University of \\ the Witwatersrand, P. O. Wits, 2050 Johannesburg, South Africa \\ e-mail: helmut@maths.wits.ac.za \\ Url: http: //www.wits.ac.za/helmut/
}

received Jan 14, 2003, accepted Aug 15, 2003.

We solve the recursion $S_{n}=S_{n-1}-q^{n} S_{n-p}$, both, explicitly, and in the limit for $n \rightarrow \infty$, proving in this way a formula due to Merlini and Sprugnoli. It is also discussed how computer algebra could be applied.

Keywords: $q$-identities, fountains, histograms, Schur polynomials

\section{Fountains and histograms}

Merlini and Sprugnoli [6] discuss fountains and histograms; for the reader's convenience, we review a few key issues here.

A fountain with $n$ coins is an arrangement of $n$ coins in rows such that each coin in a higher row touches exactly two coins in the next lower row.

A $p$-histogram is a sequence of columns in which the height of the $(j+1)$ st column is at most $k+p$, if $k$ is the height of column $j$; the first column has height $r$, with $1 \leq r \leq p$.

It can be shown that the enumeration of coins in a fountain is equivalent with the enumeration of 1-histograms. The paper [6] addresses the enumeration of $p$-histograms with respect to area (=number of cells). Let $f_{n}^{[p]}$ be the number $p$-histograms with area $n$ and $F^{[p]}(q)$ the corresponding generating function $F^{[p]}(q)=\sum_{n} f_{n}^{[p]} q^{n}$. The authors of [6] use two different approaches: one produces the answer in the form

$$
F^{[p]}(q)=\lim _{m \rightarrow \infty} \frac{D_{m}}{E_{m}}
$$

\footnotetext{
Partially supported by SFB grant F1305 of the Austrian FWF.

The research was started when this author visited the RISC in 2002. Partially supported by NRF grant 2053748.
} 
with some polynomials $D_{m}, E_{m}$ defined in the next section, and the other gives it as

$$
F^{[p]}(q)=\sum_{k \geq 0} \frac{(-1)^{k} q^{p\left(\begin{array}{c}
k+1 \\
2
\end{array}\right)}}{(1-q) \ldots\left(1-q^{k}\right)} / \sum_{k \geq 0} \frac{(-1)^{k} q^{k+p\left(\begin{array}{l}
k \\
2
\end{array}\right)}}{(1-q) \ldots\left(1-q^{k}\right)} .
$$

According to [5], it would be nice to have a direct argument that these two answers coincide. This is the subject of the present note.

\section{Generalized Schur polynomials}

The polynomials mentioned in the introduction are for fixed $p \geq 1$ defined as follows:

$$
\begin{aligned}
& E_{n}=E_{n-1}-q^{n} E_{n-p}, \quad n \geq p, \quad E_{0}=\cdots=E_{p-1}=1, \\
& D_{n}=D_{n-1}-q^{n} D_{n-p}, \quad n \geq p, \quad D_{i}=1-\sum_{j=1}^{i} q^{j}, i=0, \ldots, p-1 .
\end{aligned}
$$

They can be compared with the classical Schur polynomials [8], which occur for $p=2$ and $q=-1$. Then Merlini and Sprugnoli want a direct proof of the formulæ

$$
\begin{aligned}
& E_{\infty}:=\lim _{n \rightarrow \infty} E_{n}=\sum_{k \geq 0} \frac{(-1)^{k} q^{p\left(\begin{array}{c}
k+1 \\
2
\end{array}\right)}}{(1-q) \ldots\left(1-q^{k}\right)}, \\
& D_{\infty}:=\lim _{n \rightarrow \infty} D_{n}=\sum_{k \geq 0} \frac{(-1)^{k} q^{k+p\left(\begin{array}{c}
k \\
2
\end{array}\right)}}{(1-q) \ldots\left(1-q^{k}\right)} .
\end{aligned}
$$

We will not only achieve that but actually derive explicit expressions for these polynomials!

It should be mentioned that Cigler [4] developed independently a combinatorial method to deal with recursions as ours, but also more general ones.

Let us study the generic recursion

$$
S_{n}=S_{n-1}+t q^{n-p} S_{n-p},
$$

with unspecified initial values $S_{0}, \ldots, S_{p-1}$. For $p=2$, these polynomials were studied by Andrews (and others) in the context of Schur polynomials, see [2].

We will use standard notation from $q$-calculus, see [1]:

$$
(x)_{n}=(1-x)(1-x q) \ldots\left(1-x q^{n-1}\right), \quad\left[\begin{array}{l}
n \\
k
\end{array}\right]=\frac{(q)_{n}}{(q)_{k}(q)_{n-k}} .
$$

It will be convenient to define $\left[\begin{array}{l}n \\ k\end{array}\right]=0$ for $n<0$ or $k>n$.

Now we will proceed as in [1] and consider noncommutative variables $x$, $\eta$, such that $x \eta=q \eta x$; all other variables commute.

Lemma 1.

$$
\left(x+x^{p} \eta\right)^{n}=\sum_{k=0}^{n}\left[\begin{array}{l}
n \\
k
\end{array}\right] q^{p\left(\begin{array}{c}
n \\
2
\end{array}\right)-p n k+p\left(\begin{array}{c}
k+1 \\
2
\end{array}\right) x^{k+p(n-k)} \eta^{n-k} .}
$$


Proof. We write

$$
\left(x+x^{p} \eta\right)^{n}=\sum_{k=0}^{n} a_{n, k} x^{k+p(n-k)} \eta^{n-k},
$$

and $\left(x+x^{p} \eta\right)^{n+1}=\left(x+x^{p} \eta\right)^{n}\left(x+x^{p} \eta\right)$ resp. as $\left(x+x^{p} \eta\right)^{n+1}=\left(x+x^{p} \eta\right)\left(x+x^{p} \eta\right)^{n}$, compare coefficients, and get the recursions

$$
\begin{aligned}
& a_{n+1, k}=a_{n, k-1}+a_{n, k} q^{k+p(n-k)}, \\
& a_{n+1, k}=a_{n, k-1} q^{n+1-k}+a_{n, k} q^{p(n-k)} .
\end{aligned}
$$

From this we derive, taking differences,

$$
a_{n, k}=\frac{1-q^{n+1-k}}{1-q^{k}} q^{-p(n-k)} a_{n, k-1} .
$$

The result follows from iteration by noting that $a_{n, 0}=q^{p\left(\begin{array}{l}n \\ 2\end{array}\right)}$.

Of course we also have

$$
\left(x+t x^{p} \eta\right)^{n}=\sum_{k=0}^{n}\left[\begin{array}{l}
n \\
k
\end{array}\right] q^{p\left(\begin{array}{l}
n \\
2
\end{array}\right)-p n k+p\left(\begin{array}{c}
k+1 \\
2
\end{array}\right) x^{k+p(n-k)} t^{n-k} \eta^{n-k}} .
$$

Now we derive the generating function for

$$
F(x)=\sum_{n \geq 0} S_{n} x^{n}
$$

the following procedure is inspired by [2]. Note that we can alternatively view $\eta$ as an operator, defined by $\eta f(x)=f(q x)$. Cigler worked also much with this technique [3, 4]. We find

$$
\sum_{n \geq p} S_{n} x^{n}=\sum_{n \geq p} S_{n-1} x^{n}+\sum_{n \geq p} t q^{n-p} S_{n-p} x^{n}=x \sum_{n \geq p-1} S_{n} x^{n}+t x^{p} \sum_{n \geq 0} \eta S_{n} x^{n}
$$

or

$$
F(x)-\sum_{n<p} S_{n} x^{n}=x F(x)-x \sum_{n<p-1} S_{n} x^{n}+t x^{p} \eta F(x),
$$

and

$$
F(x)=\frac{1}{1-x-t x^{p} \eta}\left(\sum_{i<p} S_{i} x^{i}-\sum_{i<p-1} S_{i} x^{i+1}\right) .
$$

Now we can apply our lemma and write

$$
\begin{aligned}
F(x) & =\sum_{n \geq 0}\left(x+t x^{p} \eta\right)^{n}\left(\sum_{i<p} S_{i} x^{i}-\sum_{i<p-1} S_{i} x^{i+1}\right) \\
& =\sum_{n \geq 0} \sum_{k=0}^{n}\left[\begin{array}{l}
n \\
k
\end{array}\right] q^{p\left(\begin{array}{c}
n \\
2
\end{array}\right)-p n k+p\left(\begin{array}{c}
k+1 \\
2
\end{array}\right)} x^{k+p(n-k)} t^{n-k} \eta^{n-k}\left(\sum_{i<p} S_{i} x^{i}-\sum_{i<p-1} S_{i} x^{i+1}\right) \\
& =\sum_{n \geq 0} \sum_{k=0}^{n}\left[\begin{array}{l}
n \\
k
\end{array}\right] q^{p\left(\begin{array}{c}
n \\
2
\end{array}\right)-p n k+p\left(\begin{array}{c}
k+1 \\
2
\end{array}\right)} x^{k+p(n-k)} t^{n-k}\left(\sum_{i<p} S_{i} q^{i(n-k)} x^{i}-\sum_{i<p-1} S_{i} q^{(i+1)(n-k)} x^{i+1}\right)
\end{aligned}
$$




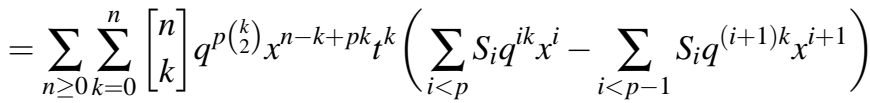

$$
\begin{aligned}
& =\sum_{k, n \geq 0}\left[\begin{array}{c}
n+k \\
k
\end{array}\right] q^{p\left(\begin{array}{c}
k \\
2
\end{array}\right) x^{n+p k} t^{k}}\left(\sum_{i<p} S_{i} q^{i k} x^{i}-\sum_{i<p-1} S_{i} q^{(i+1) k} x^{i+1}\right) \\
& =\sum_{k \geq 0} q^{p\left(\begin{array}{l}
k \\
2
\end{array}\right) x^{p k} t^{k}} \frac{1}{(x)_{k+1}}\left(\sum_{i<p} S_{i} q^{i k} x^{i}-\sum_{i<p-1} S_{i} q^{(i+1) k} x^{i+1}\right) .
\end{aligned}
$$

From this we find an explicit formula for $S_{n}$ (the quantity $S_{-1}$ has to be interpreted as 0 ):

$$
S_{n}=\sum_{0 \leq i<p}\left(S_{i}-S_{i-1}\right) \sum_{k \geq 0}\left[\begin{array}{c}
n-(p-1) k-i \\
k
\end{array}\right] q^{p\left(\begin{array}{l}
k \\
2
\end{array}\right)+i k} t^{k} .
$$

Now we specialize this to our instance. Here, $t=-q^{p}$, and thus

$$
S_{n}=\sum_{0 \leq i<p}\left(S_{i}-S_{i-1}\right) \sum_{k \geq 0}\left[\begin{array}{c}
n-(p-1) k-i \\
k
\end{array}\right] q^{p\left(\begin{array}{c}
k+1 \\
2
\end{array}\right)+i k}(-1)^{k} .
$$

Therefore

$$
E_{n}=\sum_{k \geq 0}\left[\begin{array}{c}
n-(p-1) k \\
k
\end{array}\right] q^{p\left(\begin{array}{c}
k+1 \\
2
\end{array}\right)}(-1)^{k} .
$$

From this, the limit of $E_{n}$ is immediate. For $D_{n}$ we eventually get the following form

$$
D_{n}=\sum_{k \geq 0}\left[\begin{array}{c}
n-(p-1)(k-1) \\
k
\end{array}\right] q^{k+p\left(\begin{array}{l}
k \\
2
\end{array}\right)(-1)^{k},}
$$

from which the formula for $D_{\infty}$ is immediate. To prove it, we need a simple lemma whose proof is just a routine calculation.

Lemma 2.

$$
\left[\begin{array}{c}
m-i \\
k
\end{array}\right] q^{i(k+1)}=g(i)-g(i-1) \quad \text { where } \quad g(i)=-\left[\begin{array}{c}
m-i \\
k+1
\end{array}\right] q^{(i+1)(k+1)} .
$$

Now we can plug into the general formula above and compute

$$
\begin{aligned}
& D_{n}=E_{n}-\sum_{i=1}^{p-1} \sum_{k \geq 0}\left[\begin{array}{c}
n-(p-1) k-i \\
k
\end{array}\right] q^{p\left(\begin{array}{c}
k+1 \\
2
\end{array}\right)+i(k+1)}(-1)^{k} \\
& =E_{n}-\sum_{k \geq 0}(-1)^{k} q^{p\left(\begin{array}{c}
k+1 \\
2
\end{array}\right)} \sum_{i=1}^{p-1}\left[\begin{array}{c}
n-(p-1) k-i \\
k
\end{array}\right] q^{i(k+1)} \\
& =E_{n}-\sum_{k \geq 0}(-1)^{k} q^{p\left(\begin{array}{c}
k+1 \\
2
\end{array}\right)}\left\{q^{k+1}\left[\begin{array}{c}
n-(p-1) k \\
k+1
\end{array}\right]-q^{p(k+1)}\left[\begin{array}{c}
n-(p-1)(k+1) \\
k+1
\end{array}\right]\right\} \\
& =1-\sum_{k \geq 0}(-1)^{k} q^{p\left(\begin{array}{c}
k+1 \\
2
\end{array}\right)} q^{k+1}\left[\begin{array}{c}
n-(p-1) k \\
k+1
\end{array}\right],
\end{aligned}
$$

which is the announced formula after a simple change of variable. Note that in the penultimate step the telescoping property of the lemma has been used. 


\section{Computer algebra proofs}

The polynomial families $\left(E_{n}\right)$ and $\left(D_{n}\right)$ give rise to the following study with respect to possible computer proofs. Let us take as input our sum representations of $E_{n}$ and $D_{n}$ :

$$
\begin{aligned}
& E_{n}=\sum_{k \geq 0}\left[\begin{array}{c}
n-(p-1) k \\
k
\end{array}\right] q^{p\left(\begin{array}{c}
k+1 \\
2
\end{array}\right)(-1)^{k},} \\
& D_{n}=\sum_{k \geq 0}\left[\begin{array}{c}
n-(p-1)(k-1) \\
k
\end{array}\right] q^{k+p\left(\begin{array}{l}
k \\
2
\end{array}\right)(-1)^{k} .}
\end{aligned}
$$

Then, if $p$ is chosen as a specific positive integer, Riese's package qZeil [7] returns the recurrences $S_{n}=$ $S_{n-1}-q^{n} S_{n-p}(n \geq p)$ together with a certificate function Cert for independent verification. Despite the fact that for general "generic" integer parameter $p$ there is no algorithm available, a general pattern can be easily guessed from running the algorithm for $p=1, p=2$, and $p=3$, say.

For example, let $F(n, k)$ be the $k$ th summand in our sum representation (3.1) of $E_{n}$, then the recurrence for $E_{n}$ can be refined to the following statement.

Theorem 3.1. For $n \geq p$ and $\delta_{k} f(n, k)=f(n, k)-f(n, k-1)$, we have

$$
F(n, k)-F(n-1, k)+q^{n} F(n-p, k)=\delta_{k} \operatorname{Cert}(n, k) F(n, k),
$$

where

$$
\operatorname{Cert}(n, k)=q^{n} \frac{\left(q^{n-p(k+1)+1}\right)_{p}}{\left(q^{n-(p-1)(k+1)}\right)_{p}} .
$$

Proof. After dividing both sides of 3.2 by $F(n, k)$ the proof reduces to checking equality of rational functions. Namely, note that

$$
\begin{aligned}
& \frac{F(n-1, k)}{F(n, k)}=\frac{1-q^{n-p k}}{1-q^{n-(p-1) k}}, \\
& \frac{F(n, k-1)}{F(n, k)}=-\frac{q^{p k}}{1-q^{k}} \frac{\left(q^{n-p k+1}\right)_{p}}{\left(q^{n-(p-1) k+1}\right)_{p-1}},
\end{aligned}
$$

and

$$
\frac{F(n-p, k)}{F(n, k)}=q^{-n} \operatorname{Cert}(n, k)
$$

Analogously, there is a refined version of the recurrence for $D_{n}$. The certificate in this case is

$$
\operatorname{Cert}(n, k)=q^{n} \frac{\left(q^{n-p k}\right)_{p}}{\left(q^{n-(p-1) k}\right)_{p}} .
$$

Summarizing, with the sum representation for $E_{n}$ and $D_{n}$ in hand, the corresponding recurrences follow immediately by summing both sides of the computer recurrences (3.2) over all $k \geq 0$. 


\section{References}

[1] G. E. Andrews, R. Askey, and R. Roy, Special functions, Encyclopedia of Mathematics and its Applications, vol. 71, Cambridge University Press, 1999.

[2] G. E. Andrews, Fibonacci numbers and Rogers-Ramanujan identities, The Fibonacci Quarterly, to appear (2003), 15 pp.

[3] J. Cigler, Elementare q-Identitäten, Séminaire Lotharingien de Combinatoire B05a (1981), 29 pp.

[4] J. Cigler, Some algebraic aspects of Morse code sequences, Discrete Mathematics and Theoretical Computer Science 6 (2003), 55-68.

[5] D. Merlini, Private communication, (2002).

[6] D. Merlini and R. Sprugnoli, Fountains and histograms, J. Algorithms 44 (2002), no. 1, 159-176.

[7] P. Paule and A. Riese, A Mathematica q-analogue of Zeilberger's algorithm based on an algebraically motivated approach to q-hypergeometric telescoping, Special functions, $q$-series and related topics (Toronto, ON, 1995), Fields Inst. Commun., vol. 14, Amer. Math. Soc., Providence, RI, 1997, pp. 179-210.

[8] I. Schur, Ein Beitrag zur additiven Zahlentheorie und zur Theorie der Kettenbrüche, S.-B. Preuss. Akad. Wiss. Phys.-Math. K1., 1917, 302-321, reprinted in I. Schur, Gesammelte Abhandlungen, vol. 2, pp. 117-136, Springer, 1973. 